\title{
COMPETITIVIDADE DE MARKETING EM PME EM PIRACICABA, SÃO PAULO - BRASIL
}

\author{
MARKETING competitiveness in SMEs in PIRACICABA, São PAULO-Brazil
}

\author{
Emigdio Larios - Gómez - herr.larios@gmail.com \\ PhD- Benemérita Universidad Autónoma de Puebla - BUAP - Puebla-México \\ Antonio Carlos Giuliani - giuliani.marketing@uol.com.br \\ PhD- Centro Universitário Senac- São Paulo- Brasil
}

\begin{abstract}
RESUMO
Baseado em duas investigações empíricas, que descrevem e analisam a competitividade de marketing de Micro, Pequenas e Médias Empresas (MIPMES) dos setores comercial, de serviços, industrial, construção e social no México. O primeiro integrado por empresas MIPMES na cidade de Celaya no estado de Guanajuato e o segundo por empresas MIPMES na cidade de Puebla. a pesquisa foi realizada em Micro e Pequenas Empresas MIPMES - do setor comercial e de serviços localizado na cidade de Piracicaba, no estado de São Paulo, Brasil. É uma pesquisa empírica com um não-experimental abordagem transversal e quantitativa, um questionário foi aplicado a empresas MIPMES Cidade Piracicaba no Estado de São Paulo, Brasil. A amostra foi por composta de 14 empresas (4 micros no setor comercial e 10 do setor de pequenos serviços). O objetivo foi identificar o valor do conhecimento criado pela comercialização MIPMES Brasil, por meio da transferência de conhecimento tácito e explícito e conversão do conhecimento para a competitividade de mercado. O marketing deve ser visto como um fator chave para a competitividade das empresas, como uma ferramenta estratégica para a gestão de negócios (empresas, marcas, pessoas, lugares ou produtos) que vai dar valor ao mercado, abrangendo as bases mercadológicas satisfazer as necessidades, desejos e demandas através de marketing (posicionamento, troca e venda) do seu novo produto ou não (bom, serviço, ideia ou experiência).
\end{abstract}

Palavras-chave: competitividade, gestão empresarial, marketing, MIPMES

\begin{abstract}
Based on two empirical investigations that describe and analyze the marketing competitiveness of Micro, Small and Medium Enterprises (MISMES) of the commercial, services, industrial, construction and social sectors in Mexico. The first is composed of MISMES companies in the city of Celaya in the state of Guanajuato and the second by MISMES companies in the city of Puebla. the survey was conducted in Micro and Small Businesses - MIPES - of the commercial and services sector located in the city of Piracicaba, in the state of São Paulo, Brazil. It is an empirical research with a nonexperimental cross-sectional and quantitative approach, a questionnaire was applied to companies MISMES Cidade Piracicaba in the State of São Paulo, Brazil. The sample consisted of 14 companies (4 in the commercial sector and 10 in the small services sector). The objective was to identify the value of knowledge created by MISMES Brasil commercialization, through the transfer of tacit and explicit knowledge and the conversion of knowledge to market competitiveness. Marketing should be seen as a key factor for the competitiveness of companies, as a strategic tool for business management
\end{abstract}


(companies, brands, people, places or products) that will give value to the market, covering the market bases meet the needs, desires and demands through marketing (positioning, exchange and sale) of your new product or not (good, service, idea or experience)

Keywords: competitiveness, business management, marketing, MISMES

\section{DOI:}

\section{INTRODUÇÃO}

Baseado em duas investigações empíricas, que descrevem e analisam a competitividade de marketing de Micro, Pequenas e Médias Empresas (MIPMES) dos setores comercial, de serviços, industrial, construção e social no México. O primeiro integrado por empresas MIPMES na cidade de Celaya no estado de Guanajuato (LARIOS-GÓMEZ, 2016a) e o segundo por empresas MIPMES na cidade de Puebla (LARIOS-GÓMEZ, 2016b).

A pesquisa deste artigo foi realizada em MIPMES do setor comercial e de serviços localizado na cidade de Piracicaba, no Estado de São Paulo, Brasil, em que a Gestão do Conhecimento nas organizações (DAVENPORT; PRUSAK, 2001) é descrita como uma disciplina estratégica de processos inteligentes (lógicos, organizados e sistemáticos), que promove a geração, colaboração, interação, transferência e uso de conhecimento explícito e tácito na conversão de uma expertise (mistura de conhecimentos, experiências, valores e informações dos indivíduos), para o aprendizado organizacional de uma unidade econômica, gerando novo valor de competitividade para atingir seus objetivos de forma eficiente e eficaz.

No que diz respeito à competitividade das MIPMES deve ser entendido como a capacidade de projetar, criar, produzir, oferecer, distribuir e comercializar produtos (bens, serviços, ideias ou experiências) valor inovador e de alto valor acrescentado superior aos oferecidos no mercado, nas condições, quantidade e qualidade que o cliente (pessoalmente ou virtual), demanda nacional e/ou internacional. Destacando nesta relação, a capacidade competitiva deste tipo de empresas: 1) como elas gerenciam seus conhecimentos e 2) a relação desse conhecimento com o seu desempenho no mercado (DINI; STUMPO, 2004; LARIOS-GÓMEZ, 2016b). Ou seja, marketing, e de uma perspectiva de gestão do conhecimento, o marketing é uma ferramenta estratégica de gestão empresarial (empresas, marcas, pessoas, lugares ou produtos), que nos permite 
criar valor para atender necessidades, desejos e demandas, através da comercialização (posicionamento, troca e venda) de um novo produto ou não (bem, serviço, ideia ou experiência) direta ou indiretamente (presencial ou virtual), com base na pesquisa de informações e experiências coletadas de um mercado específico (clientes, consumidores, funcionários, concorrentes, fornecedores e parceiros) para a competitividade da empresa.

Para Gyulavári e Kenesei (2012), o marketing é um fator de sucesso para a competitividade de uma empresa, enfatizando que os ativos e as capacidades de marketing estão intimamente relacionados ao desempenho dos negócios. E as empresas competitivas são aquelas que têm melhores capacidades de marketing e têm uma vantagem competitiva na personalização flexível de produtos e na entrega rápida e precisa.

Por outro lado, com base em Castellanos (2003), existem dois tipos de abordagem à gestão do conhecimento de estratégias de marketing em MIPMES: estratégias de sobrevivência, que tentam manter a lucratividade e o progresso atuais da empresa, que garantem a rentabilidade futura da organização (sustentabilidade). Finalmente, retornando a Bozbura (2007), pode-se dizer que o Marketing Knowledge Management possui quatro dimensões essenciais: 1) treinamento de marketing em funcionários, 2) políticas e estratégias comerciais, 3) criação e aquisição de conhecimento de marketing ( externos e internos) e 4) efeitos da cultura organizacional de marketing.

\section{COMPETITIVIDADE NO MIPMES}

As Micro Pequenas e Médias Empresas (MIPMES) latinoamericanas só podem sobreviver no processo de desenvolvimento industrial se produzirem bens e serviços específicos que não competem com os produtos produzidos em massa de grandes empresas industriais (OECD, 2010).

Normalmente, são produtos projetados de acordo com as necessidades do cliente, pequenas séries para nichos de mercado ou produtos intimamente relacionados ao atendimento ao cliente (instalação, adaptação específica às suas necessidades, manutenção e outros). Essas PMEs também produzem serviços para grandes empresas ou complementam sua oferta de serviços.

Atualmente, a especialização da empresa, impõe maiores exigências de qualificação técnica e empresarial, sendo condição prévia, em especial, a capacidade de inovar continuamente. A maioria das PMEs na América Latina não possui essas qualificações. Quase todas essas empresas estão inseridas em áreas padronizadas de 
produção com baixa intensidade de conhecimento, onde competem diretamente com produção em grande escala e / ou com grandes casas comerciais. Um exemplo disso é a produção de bens de consumo de massa (alimentos genéricos, calçados, roupas), nos quais as PMEs latinoamericanas não encontram vantagens competitivas (ALTENBURG; ECKHARDT 2006).

Como pode ser visto na Figura 1, as PMEs nos países desenvolvidos funcionam como apoio e fornecem para grandes empresas e outras oferecem produtos e serviços personalizados para não competir com os grandes. Caso contrário, isso acontece em PMEs em países em desenvolvimento (como a América Latina), onde a maioria das PMEs competem em mercados de produtos em massa, amontoados por grandes empresas e o resultado disso é um círculo vicioso em que a baixa A produtividade inicial e a falta de transferência de conhecimento entre as empresas reforçam-se mutuamente (ALTENBURG, 2011).

Figura 1 - Organização Industrial das PME

Típica organización industrial en países desarrollados

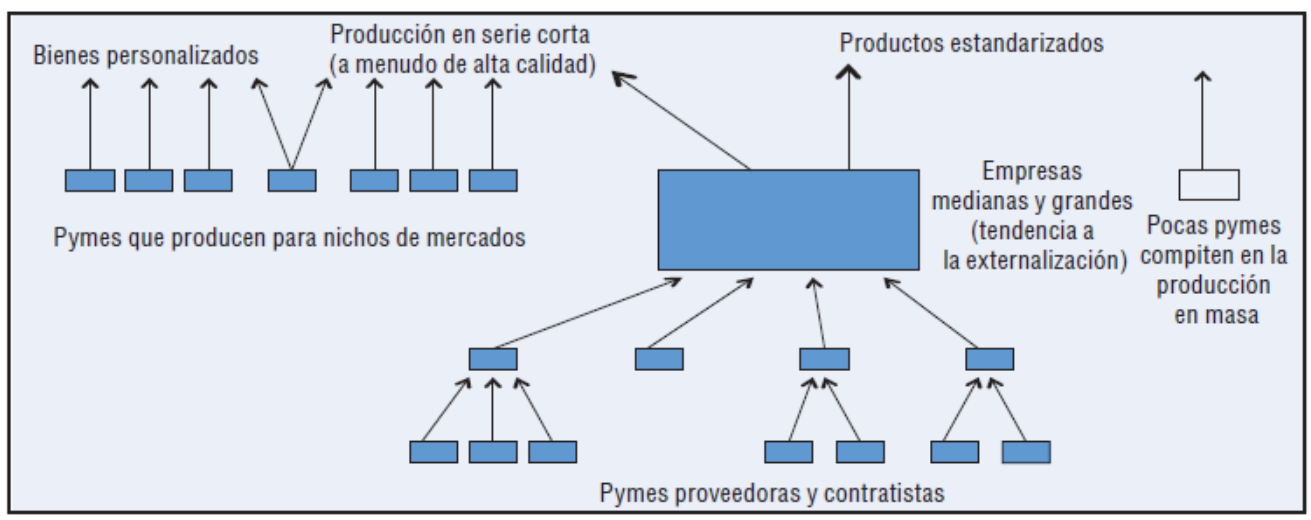

Típica organización industrial en países en desarrollo

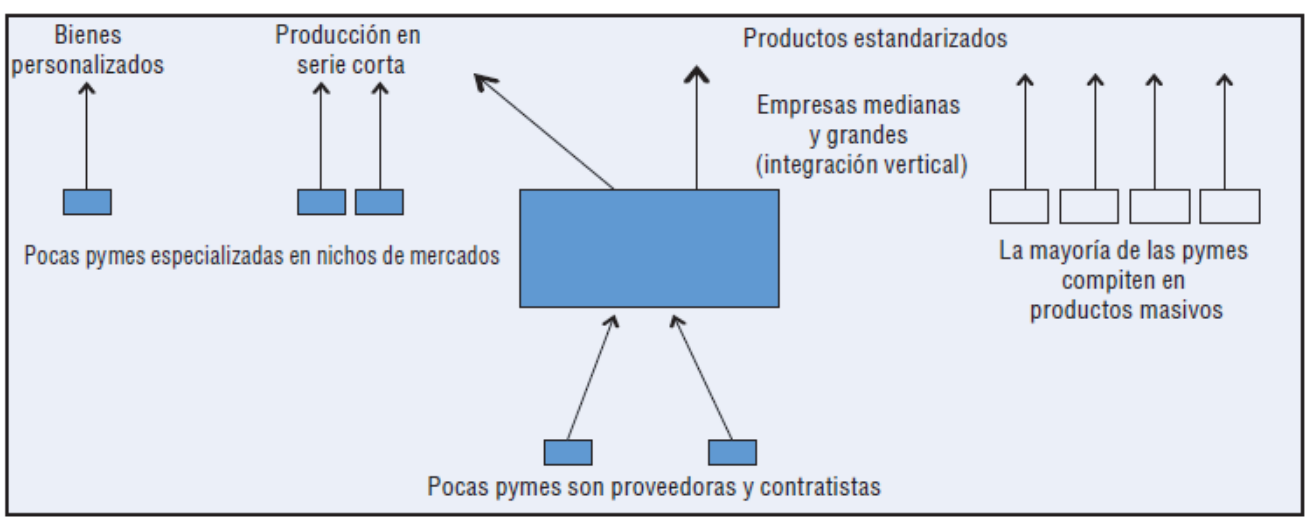

Fonte: Altenburg e Eckhardt (2006). 
No México existem cerca de quatro milhões de empresas, destes, apenas 6.700 são grandes empresas, o micro resto, pequenas e médias empresas representam 99,8\%, geram $52 \%$ do produto interno bruto (PIB) e contribuir $72 \%$ dos postos de trabalho formal Microempresa: representam 95\% das empresas estabelecidas e 38,3\% do emprego, muitos não gerar lucros ou têm níveis negativos da produtividade e a sua contribuição para o PIB é muito fraco, por isso, é necessário implementar uma política que visa alcançar que são reconhecidos como negócios que devem gerar riqueza. Enquanto as pequenas e médias empresas: eles representam 4,8\% das empresas do país e gerar 32,7\% do emprego, são altamente produtivas e são a espinha dorsal da atividade econômica nacional a ser os principais fornecedores de grandes empresas (OCDE; FOUNDATION IDEA, 2010).

Apesar de sua importância numérica e ao contrário de empresas maiores, as MPME mexicanas têm que superar vários obstáculos ao seu desenvolvimento e sobrevivência. MPMEs enfrentam um difícil acesso ao financiamento, comercializa pouca informação sobre os seus produtos, capital humano com pouco treinamento, tecnologia limitada e ignorância sobre os requisitos e normas para comercializar os seus produtos nos mercados internacionais usam.

Para definir a competitividade das empresas, com base no mencionado por Perez e Perez (2008) conceituação de competitividade foi modificado tanto na sua definição e conteúdo de acordo com as perspectivas da economia global. Focando seu objetivo nas esferas nacional, setorial e empresarial. Para esse mesmo autor, competitividade é a capacidade de uma organização pública ou privada de manter vantagens que lhe permitam alcançar e melhorar sua posição socioeconômica com outras empresas.

Além disso Cruz (2012) menciona que as empresas envolvidas na produção de bens deve buscar a inovação para ser competitivo, mas muitos são prejudicados este trabalho para processos de negócios e sistemas informáticos inadequados, que geram falhas de comunicação, erros trabalho, atrasos e custos mais elevados.

De la Cruz, Morales e Carrasco (2006) argumentam que a capacidade num sector e as suas empresas contribuam para aumentar a competitividade de uma gama de produtos ou serviços, eles também influenciam profundamente as capacidades de crescimento e diferenciação competitiva a partir de uma gama de produtos muito além do sucesso ou fracasso de um único produto.

Solleiro e Castañón (2005) argumentam que a competitividade é um conceito complexo que tem sido estudado a partir de diferentes abordagens e disciplinas. Eles 
apontam que o desempenho competitivo da empresa depende principalmente de sua capacidade de gerenciar elementos internos estão sob controle, complementar a este ponto que a competitividade depende também da qualidade das interações que a empresa estabeleceu uma série de fatores internos e externos. Nos contribuições destes autores, os elementos que influenciam a competitividade das empresas são: o êxito da gestão dos fluxos de produção, matérias-primas e de inventário, a gestão bem sucedida dos mecanismos de interação entre o planejamento, marketing, pesquisa e desenvolvimento formal, design, engenharia e produção industrial, a capacidade de combinar pesquisa e desenvolvimento e inovação em cooperação com universidades e outras empresas, a capacidade de incorporar definiçõos mais precisas das características da demanda e evolução do mercado na concepção e produção de estratégias, a capacidade de organizar com sucesso as relações entre empresas com fornecedores e clientes e a melhoria das capacidades dos trabalhadores através do investimento em treinamento especializado e a geração de altos níveis de responsabilidade nos trabalhadores da produção.

\subsection{Contexto econômico da cidade de Piracicaba, São Paulo, Brasil}

A cidade é um importante pólo regional de desenvolvimento industrial e agrícola, estando localizada em uma das regiões mais industrializadas e produtivas de todo o Estado de São Paulo. A região concentra uma população aproximada de 1,2 milhão de habitantes (IVACE, 2015). Uma das principais cidades do Estado de São Paulo, às margens do rio Piracicaba, era vital para a região. Durante o século, a agricultura foi desenvolvido no município, com destaque para o cultivo de cana-de-açúcar e café, para o ciclo do café e a queda constante dos preços de açúcar de cana, a economia Piracicabana estagnou. Este foi revertida a partir do momento em que a cidade se tornou um dos primeiros a industrializar o país, com a abertura de fábricas ligadas a equipamentos metalmecânico e de produção para o setor de açúcar.

A cidade é o lar de um dos principais centros industriais da região, além de várias universidades de renome, como a Escola Superior de Agricultura Luiz de Queiroz (ESALQ), pertencente à Universidade de São Paulo (USP), a Faculdade de Odontologia de Piracicaba, pertencente à Universidade Estadual de Campinas (UNICAMP) e da Universidade Metodista de Piracicaba (UNIMEP) (IVACE, 2015). O complexo industrial da região de Piracicaba é composto por mais de cinco mil indústrias, com destaque para 
as atividades da metalúrgica, mecânica, têxtil, alimentos e combustível (produção de petroquímicos e álcool) setores.

A questão de pesquisa é: "se o marketing é a chave para a competitividade das pequenas e grandes empresas nos Estados Unidos, Inglaterra, Alemanha ou fator de Espanha. Então, o MIPES brasileiro pode ser competitivo do ponto de vista de marketing?" A Gestão de Marketing a partir da perspectiva do planejamento estratégico é um fator chave para a competitividade da CPDM no Brasil?

\section{MÉTODO}

É uma pesquisa empírica de abordagem quantitativa (KERLINGER, 2002; MALHOTRA, 2009). Foi aplicado um questionário para as MIPES, da cidade de Piracicaba, no Estado de São Paulo, Brasil. A amostra foi por conveniência de 14 empresas (4 micros no setor comercial e 10 do setor de pequenos serviços).

O objetivo foi identificar o valor do conhecimento criado pela comercialização MIPES Brasil, por meio da transferência de conhecimento tácito e explícito e conversão do conhecimento para a competitividade de mercado. Os ciclos modelo de Gestão do Conhecimento Probst, Raub e Romhardt (2001) foi tomada porque contempla aspectos estratégicos das organizações, além de dar maior ênfase à medição e avaliação de conhecimento que também serve como feedback para a organização interessados na gestão do seu conhecimento (Perez-Soltero et al., 2013), para o qual o modelo foi adaptado a partir de uma perspectiva de marketing em questões como planejamento, cultura organizacional e Uso de Tecnologia (LARIOS GÓMEZ, 2016a e 2016b).

O estudo levou em conta o tamanho da empresa de acordo com a categoria do Serviço Brasileiro de Apoio às Micro e Pequenas Empresas (Sebrae pela sigla em Português) é uma entidade privada que promove a competitividade e o desenvolvimento uso sustentável das microempresas e pequenas dimensões (SEBRAE, 2013), que considera o número de empregados (Quadro 1). 
Quadro 1 - Classificação das MPMEs no Brasil.

\begin{tabular}{|c|c|c|c|}
\hline \multicolumn{4}{|c|}{$\begin{array}{l}\text { Definición de las Mic } \\
\text { Empresas en Brasil }\end{array}$} \\
\hline $\begin{array}{c}\text { Porte de las } \\
\text { empresas } \\
\text { Ordenacion Juridica }\end{array}$ & Micro Empresa & Pequena Empresa & Modiana Empresa \\
\hline $\begin{array}{l}\text { Estatuto de la } \\
\text { MiprMES } \\
\text { Facturacion bruta } \\
\text { anual }\end{array}$ & RS $433.000,00$ & RS 2.133.000,00. & $-m$ \\
\hline $\begin{array}{c}\text { SIMPLES } \\
\text { Facturacion bruta } \\
\text { anual }\end{array}$ & Rs $240,000,00$ & RS $2.400 .000,00$ & $\longrightarrow$ \\
\hline $\begin{array}{c}\text { MTERRAIS } \\
\mathrm{N}^{0} \text { de empleados }\end{array}$ & $0-19$ & $20 \cdot 9$ & $100-499$ \\
\hline $\begin{array}{c}\text { SEBRAE } \\
\text { Industria } \\
N^{0} \text { de empleados }\end{array}$ & $0-19$ & $20-99$ & $100-499$ \\
\hline $\begin{array}{c}\text { SEBRAE } \\
\text { Comercio y Servicios } \\
\mathrm{N}^{5} \text { de empleados }\end{array}$ & $0-9$ & $10-49$ & $50-99$ \\
\hline
\end{tabular}

Fonte: SEBRAE (2013).

\section{RESULTADOS}

Planejamento. O MIPES do setor de comércio de Piracicaba, São Paulo-Brasil não realiza de forma conclusiva o planejamento das atividades de marketing. Como pode ser visto na Tabela 1, não há interesse em desenvolver a missão, visão, objetivos e planejamento de marketing, se existe, em termos gerais (média de 2,98), não é favorável completamente, uma vez que também Pode-se dizer que esses MIPES não realizam planejamento nas atividades de marketing. E os MIPES do setor de serviços têm um interesse relativamente maior, porque eles têm um plano estratégico de marketing (juros em média de 3,10). A concepção, desenvolvimento e implementação do planejamento estratégico de marketing começa com a existência da missão de marketing que se baseia na missão da empresa, assim como na visão e objetivos do marketing. Pode-se dizer que 
estes MIPES não realizam planejamento nas atividades de marketing por completo, apesar de terem planos para o posicionamento e melhoria de seus serviços.

Cultura Organizacional. No aspecto da cultura organizacional de Marketing, os resultados são mais encorajadores, isto é, embora o setor MIPES comércio no MIPES de Piracicaba, São Paulo-Brasil, a falta de planejamento de marketing, se eles estão cientes do impacto atual de estratégias de marketing e conhecimento nas empresas, ainda mais no seu. A média para este construto é de 3,99, essas empresas estão cientes da importância de planejar o impacto que o marketing pode ter em sua cultura organizacional, por meio de manuais, comunicação interna e do clima que é gerado na empresa. E os MIPES do setor de serviços apresentam uma melhor cultura organizacional de marketing do que as empresas do setor de serviços, com uma média de 4,52. Pode-se denotar a preocupação que impacta o ambiente organizacional da empresa nas decisões de marketing, principalmente quando a essência ou objeto de atuação das empresas está relacionado a produtos intangíveis, como serviços e missão social. Assim, deduzimos que essas empresas estão quase completamente interessadas, preocupadas e ocupadas em ter um ambiente de trabalho agradável e comunicação eficiente entre os membros da empresa, aprimorando as ferramentas de marketing.

Tecnologia. No que diz respeito à tecnologia para o desenvolvimento e implementação de estratégias de marketing, o MIPES do setor de comércio da cidade de Piracicaba, se usar, possuir ou investir em tecnologia como PCs, impressoras, acesso à internet, códigos de barra, circuitos fechados, ou softwares para a administração de clientes, sites, telemarketing, uso de e-mails, na perspectiva do marketing. A média de 4,90, podemos dizer que, além de preocupados, são ocupados por dispor das ferramentas tecnológicas necessárias para a competitividade no mercado, contando com terminais de coleta e uso de plataformas eletrônicas com seu respectivo uso de e-commerce. E o MIPES do setor de serviços, (média de 4,05), faz uso de ferramentas tecnológicas para a competitividade de suas operações. Através de software livre e comercial, como plataformas CMR e Telemarketing.

Tabela 1 - Gestão de Marketing no Planejamento Estratégico do MIPES Brasil

\begin{tabular}{l|l}
\hline Variable & Piracicaba, São Paulo -Brasil
\end{tabular}




\begin{tabular}{l|cc}
\hline & Comercio & Servicios \\
\cline { 2 - 3 } Estrategia & 2.98 & 3.10 \\
Cultura Organizacional & 3.99 & 4.52 \\
Tecnología & 4.90 & 4.05 \\
\hline
\end{tabular}

Fonte: Elaboração própria

\section{CONCLUSÃO}

Os MIPMES da América Latina, no México, Chile, Argentina ou Brasil, quase sempre compartilham as mesmas características, o mais destacado da abordagem de GC é a gestão empírica e sem expertise do setor para ser competitiva do ponto de vista de marketing, já que a falta de planos ou programas estratégicos nesta disciplina faz com que eles desapareçam ou tenham problemas em seus processos de marketing e posicionamento.

O marketing pode ser usado por qualquer tipo de negócio, independentemente do seu bouquet ou tamanho. No entanto, a realidade é que as micro ou pequenas empresas não possuem recursos ou experiência para explorar o marketing em suas formas mais sofisticadas, aplicam o marketing de maneira diferente e são baseadas em decisões intuitivas e são reafirmadas por este estudo.

O marketing deve ser visto como um fator chave para a competitividade das empresas. Como uma ferramenta estratégica para a gestão de negócios (empresas, marcas, pessoas, lugares ou produtos) que vai dar valor ao mercado, abrangendo as bases mercadológicas satisfazer as necessidades, desejos e demandas através de marketing (posicionamento, troca e venda) do seu novo produto ou não (bom, serviço, ideia ou experiência).

Nos diferentes canais de marketing, sem altos investimentos, direta ou indiretamente (ou virtual) forma porque recolhe, analisa, sintetiza e gerencia informações de pesquisas e experiências coletadas de um mercado específico (clientes, consumidores, empregados, concorrentes, fornecedores e parceiros) para a competitividade da empresa.

\section{REFERÊNCIAS}

ALTENBURG, T. Industrial Policy in Developing Countries. Overview and lessons from seven country cases. Discussion Paper 4/11, German Development Institute, Bonn. 2011. 
.; ECKHARDT, U. Productivity Enhancement and Equitable Development: Challenges for SME Development, UNIDO, Viena. 2006.

CRUZ, A. (2012), Pequeñas empresas. Innovación indispensable para empresas de manufactura, 2012. Disponível em:

<http://www.pymempresario.com/2012/02/innovacion- indispensable-para-empresasdemanufactura $>$. Acesso em 08 set. 2018.

DAVENPORT, T. H.; Prusak, L. Conocimiento en acción. Cómo las organizaciones manejan lo que saben. Buenos Aires: Prentice Hall. 2001.

DE LA CRUZ; MORALES; CARRASCO (2006). Determinación de la competitividad en la PYME Latinoamericana, Revista de investigación Institucional, 2006. pp. 3. Disponível em: <http://www.alafec.unam.mx/docs/pymes.pdf>. Acesso em: 08 set. 2018.

DINI, M. Y G.; STUMPO (COORDS.) Pequeñas y medianas empresas y eficiencia colectiva. Estudios de caso en América Latina, CEPAL-Siglo XXI Editores, México, D.F. 2004

IVACE. Oficina Económica y Comercial de España en Brasilia. 2015.

Kerlinger. Investigación del Comportamiento, McGraw Hill: México. 2002.

LARIOS-GOMEZ, E. La Gestión de la Competitividad en la MIPYME Mexicana: Diagnóstico Empírico desde la Gestión del Conocimiento. Revista de Administração da Unimep, 14(2). 2016a.

. La competitividad de mercadotecnia y la gestión del conocimiento en la mipyme del sector retailing en Celaya, México. Revista de Administração, Contabilidade e Economia da Fundace. 8(1). 2016 b.

MALHOTRA, N. (2009). Investigación de mercados. 5. E. Pearson: México. 2009.

OCDE. Entrepreneurship at a Glance 2012, OCDE, París.

OECD; Fundación IDEA. Consolidación de la competencia económica y la mejora regulatoria para la competitividad en México, Estudio de caso. Puebla, Prácticas y políticas exitosas para la mejora regulatoria y el emprendedurismo a nivel subnacional. México. 2010.

PÉREZ, A.; PÉREZ, O. Competitividad y acumulación de capacidades tecnológicas en la industria manufacturera mexicana. Universidad Autónoma de Zacatecas. 2008. Disponível em:

<http://redalyc.uaemex.mx/src/inicio/ArtPdfRed.jsp?iCve=60111154005.>. Acesso em: 08 set. 2018.

PEREZ-SOLTERO, A.; LEAL SOTO, V.; BARCELÓ VALENZUELA, M.; LEÓN DUARTE, J. A. 2013. Un diagnóstico de la gestión del conocimiento en las pymes 
del sector restaurantero para identificar áreas de mejora en sus procesos productivos. Intangible Capital, 9(1).

PROBST, G.; RAUB S.; ROMHARDT, K.. Administre el Conocimiento: Los Pilares del éxito. Traducido del (Ingles) por Núñez A. México: Pearson Educación. 2001.

SEBRAE -Serviço Brasileiro de Apoio às Micro e Pequenas Empresas. Pequenos Negócios. Desafios e Perspectivas Sebrae. 2013. Vol. 5. Disponível em: <http://www.bibliotecas.sebrae.com.br/chronus/ARQUIVOS_CHRONUS/bds/bds.nsf/b 449b8c1b93575d03b773ffadfc757bc/\$File/4508.pdf>. Acesso em: 08 set. 2018.

SOLLEIRO; CASTAÑÓN. Competitividad y sistemas de Innovación: Los retos para la inserción de México en el Contexto Global. Revista Iberoamericana, 2005. vol. 5, pp. 15. Disponível em: <http://www.oei.es/salactsi/solleiro.pdf.>. Acesso em: 08 set. 2018. 